\title{
ANOSOV DIFFEOMORPHISMS ON TORI
}

\author{
BY \\ JOHN FRANKS $\left({ }^{1}\right)$
}

An Anosov diffeomorphism $f: M \rightarrow M$ is a diffeomorphism which satisfies the following:

(a) There is a continuous splitting of the tangent bundle $T M=E^{s}+E^{u}$ which is preserved by the derivative $d f$.

(b) There exist constants $C>0, C^{\prime}>0$ and $\lambda \in(0,1)$ and a Riemannian metric $\|\quad\|$ on $T M$ such that

and

$$
\left\|d f^{n}(v)\right\| \leqq C \lambda^{n}\|v\| \quad \text { for } v \in E^{s}
$$

$$
\left\|d f^{n}(v)\right\| \geqq C^{\prime} \lambda^{-n}\|v\| \quad \text { for } v \in E^{u} .
$$

In [7] S. Smale poses the problem of classifying all Anosov diffeomorphisms of compact manifolds up to topological conjugacy. The purpose of this paper is to consider this problem in the special case that $M$ is the $n$ dimensional torus $T^{n}=S^{1} \times \cdots \times S^{1}$.

Examples of Anosov diffeomorphisms on $T^{n}$ can be constructed as follows. Let $\bar{f}$ be an $n \times n$ matrix with integer entries and no eigenvalues of absolute value one. The isomorphism of $R^{n}$ defined by $\bar{f}$ maps the integer lattice $J$ of $R^{n}$ to itself and hence induces an automorphism $f$ of $R^{n} / J=T^{n}$. $f$ is easily shown to be an Anosov diffeomorphism. We shall call examples constructed in this way hyperbolic toral automorphisms.

To study an arbitrary Anosov diffeomorphism $f: T^{n} \rightarrow T^{n}$, we will need the additional hypothesis that the nonwandering set of $f, N W(f)$ (see [7] for definition) is the entire torus $T^{n}$. It seems a reasonable conjecture that all Anosov diffeomorphisms satisfy this condition. It will certainly hold if there is a Lebesgue measure on $T^{n}$ which is invariant under $f$.

THEOREM 1. If $f: T^{n} \rightarrow T^{n}$ is an Anosov diffeomorphism with $N W(f)=T^{n}$ and if $f_{*}: H_{1}\left(T^{n}, R\right) \rightarrow H_{1}\left(T^{n}, R\right)$ is hyperbolic (i.e. has no eigenvalues of absolute value 1$)$, then $f$ is topologically conjugate to a hyperbolic toral automorphism.

A somewhat weaker version of this theorem for Anosov diffeomorphisms of the two dimensional torus was proved by A. Avez in [1]. A slightly more general result for dimensions two and three is proven in [3].

Received by the editors March 10, 1969.

( ${ }^{1}$ This research was partially supported by the Air Force Office of Scientific Research, Contract No. F44620-67-C-0029. 
In view of the hypothesis of Theorem 1 it is of interest to know what homotopy classes admit Anosov diffeomorphisms. It seems reasonable to conjecture that for any Anosov diffeomorphism $f: T^{n} \rightarrow T^{n}, f_{*}: H_{1}\left(T^{n}, R\right) \rightarrow H_{1}\left(T^{n}, R\right)$ is hyperbolic. Toward this end we prove

THEOREM 2. If $f: T^{n} \rightarrow T^{n}$ is an Anosov diffeomorphism then $f_{*}: H_{1}\left(T^{n}, R\right) \rightarrow$ $H_{1}\left(T^{n}, R\right)$ has no eigenvalues which are roots of unity.

1. Hyperbolic Anosov diffeomorphisms. The main lemma used in proving Theorem 1 is

(1.1) LeMma. If $f: T^{n} \rightarrow T^{n}$ is an Anosov diffeomorphism with fixed point $x_{0}$ and $f_{*}: H_{1}\left(T^{n}, R\right) \rightarrow H_{1}\left(T^{n}, R\right)$ is hyperbolic then there exists a hyperbolic toral automorphism $g: T^{n} \rightarrow T^{n}$ and a map $h: T^{n} \rightarrow T^{n}$ homotopic to the identity such that $h\left(x_{0}\right)=0$ and $g \circ h=h \circ f$.

Proof. This is an immediate corollary to (2.1) of [3] or Theorem 1 of [4].

(1.2) LEMMA. If $f: T^{n} \rightarrow T^{n}$ is an Anosov diffeomorphism and $f_{*}: H_{1}\left(T^{n}, R\right) \rightarrow$ $H_{1}\left(T^{n}, R\right)$ is hyperbolic then a lifting $\bar{f}: R^{n} \rightarrow R^{n}$ has exactly one fixed point.

Proof. Let $g: T^{n} \rightarrow T^{n}$ be the automorphism of the torus such that

$$
f_{*}=g_{*}: H_{1}\left(T^{n}, R\right) \rightarrow H_{1}\left(T^{n}, R\right) .
$$

$g$ is a hyperbolic toral automorphism. If $\bar{g}: R^{n} \rightarrow R^{n}$ is the linear map covering $g$ then it follows easily that $\bar{g}$ is a finite distance from $\bar{f}$; that is, there exists a finite $K$ such that $K=\sup _{x \in R^{n}}\|\bar{f}(x)-\bar{g}(x)\|$.

Since $\bar{g}$ is hyperbolic $\bar{g}-I$ is an isomorphism of $R^{n}$ ( $I$ is the identity map on $R^{n}$ ). Let $\mu=\inf _{\|x\|=1}\|(\bar{g}-I)(x)\|$ and choose $r>0$ such that $\mu r>K$.

$\bar{g}$ has the single fixed point 0 and the index of this fixed point (see [2]) can be defined by $(I-\bar{g})_{*}(1)$ where 1 is a generator of $H_{n}\left(R^{n}, R^{n}-0\right)$ and

$$
(I-\bar{g})_{*}: H_{n}\left(R^{n}, R^{n}-0\right) \rightarrow H_{n}\left(R^{n}, R^{n}-0\right)
$$

is the homomorphism induced by $(I-\bar{g})$. If we denote this index by $i_{g}(0)$, it follows easily from the properties of the index (see [2]) and the fact that $\bar{g}$ is hyperbolic that $i_{g}(0)= \pm 1$ (the sign is not important for our purposes).

Note that if $\|x\|>r$ then

$$
\|x-\bar{f}(x)\| \geqq\|x-\bar{g}(x)\|-\|\bar{g}(x)-\bar{f}(x)\| \geqq \mu r-K>0,
$$

so any fixed points of $\bar{f}$ are contained in the ball of radius $r$. Moreover $\bar{f}$ is an Anosov diffeomorphism so the fixed points must be isolated. Hence there can be only a finite number of them. Let $F=\{x \mid \bar{f}(x)=x\}$ then as before we define $i_{f}(F)=(I-\bar{f})_{*}(e)$ where $(I-\bar{f})_{*}: H_{n}\left(R^{n}, R^{n}-F\right) \rightarrow H_{n}\left(R_{n}, R^{n}-0\right)$ and $e$ is the image of 1 under the composite map

$$
Z=H_{n}\left(S^{n}\right) \rightarrow H_{n}\left(S^{n}, S^{n}-F\right)=H_{n}\left(R^{n}, R^{n}-F\right) .
$$


Let $k:[0,1] \times R^{n} \rightarrow R^{n}$ be given by $k(t, x)=t \bar{g}(x)+(1-t) \bar{f}(x) . k$ is a homotopy between $\bar{f}$ and $\bar{g}$ and if $\|x\|>r$ then

$$
\|x-k(t, x)\| \geqq\|x-\bar{g}(x)\|-\|\bar{g}(x)-k(t, x)\| \geqq \mu r-K>0
$$

so for any $t, k(t, \quad)$ has no fixed points outside the ball of radius $r$. Consequently by (1.7) of [2], $i_{g}(0)=i_{f}(F)$.

On the other hand from (1.5) of [2] we have $i_{f}(F)=\sum_{x \in F} i_{f}(x)$. If $x \in F$ then it is a hyperbolic fixed point of $\bar{f}$. Consequently $i_{f}(x)= \pm 1$, the sign depending on the dimension of $E^{u}$ and whether or not $d \bar{f}_{x}: E_{x}^{u} \rightarrow E_{x}^{u}$ reverses orientation. Since $R^{n}$ is simply connected, the bundle $E^{u}(\bar{f}) \rightarrow R^{n}$ is orientable so $d \bar{f}$ either preserves orientation everywhere or reverses it everywhere. Consequently for all $x \in F, i_{f}(x)$ has the same sign. If $N(\bar{f})$ denotes the number of fixed points of $\bar{f}$ then

$$
N(\bar{f})=\left|\sum_{x \in F} i_{f}(x)\right|=\left|i_{f}(F)\right|=\left|i_{g}(0)\right|=1 .
$$

(1.3) COROLlary. If $f: T^{n} \rightarrow T^{n}$ is an Anosov diffeomorphism and

$$
f_{*}: H_{1}\left(T^{n}, R\right) \rightarrow H_{1}\left(T^{n}, R\right)
$$

is hyperbolic then $f$ has a fixed point.

Proof. If $P: R^{n} \rightarrow T^{n}$ is the covering map and $x_{0}$ is the fixed point of $\bar{f}: R^{n} \rightarrow R^{n}$ then $P\left(x_{0}\right)$ is a fixed point for $f$. Q.E.D.

If $f: T^{n} \rightarrow T^{n}$ is an Anosov diffeomorphism then the stable and unstable manifolds of $f$ (see [5]) form two everywhere transverse foliations of $T^{n}$. These foliations can be lifted to $R^{n}$ giving a foliation $U$ each leaf of which covers an unstable manifold of $f$ and a foliation $S$ covering stable manifolds. Each leaf of $U(S)$ is a $k$-plane ( $n-k$ plane) where $k=\operatorname{dim} E^{u}$. If $u(x)$ denotes the leaf of $U$ passing through $x$ then $u(x)=u(y)$ iff $\lim _{n \rightarrow-\infty}\left\|\bar{f}^{n}(x)-\bar{f}^{n}(y)\right\|=0$, and similarly if $s(x)$ denotes the leaf of $S$ passing through $x$ then $s(x)=s(y)$ iff $\lim _{n \rightarrow \infty}\left\|\bar{f}^{n}(x)-\bar{f}^{n}(y)\right\|=0$. These and other basic properties of $S$ and $U$ are proved in $\S 1$ of [3].

If $W^{u}(x)$ denotes the unstable manifold of $x$, let $W^{u}(x ; \varepsilon)=$ the component of $x$ in $\left\{y \mid y \in W^{u}(x)\right.$ and $\left.|y-x|<\varepsilon\right\}$. Define $W^{s}(x ; \varepsilon)$ similarly. If $N W(f)=T^{n}$, then from (7.4) of [7] we have

(1.4) Lemma (Local Product Structure). There is an $\varepsilon>0$ and an embedding $j: W^{u}(x ; \varepsilon) \times W^{s}(x ; \varepsilon) \rightarrow T^{n}$ which is given by $j\left(x_{1}, x_{2}\right)=$ the unique point of intersection of $W^{u}\left(x_{1} ; \varepsilon\right)$ and $W^{s}\left(x_{2} ; \varepsilon\right) . \varepsilon$ is independent of $x$.

If $N$ is the image of $j, N$ will be called a product neighborhood of $x$. If $N$ is sufficiently small it can be lifted to a product neighborhood in $R^{n}$.

(1.5) Lemma. If $f: T^{n} \rightarrow T^{n}$ is an Anosov diffeomorphism with $N W(f)=T^{n}$ and if $u$ and $s$ are leaves of the induced foliations $U$ and $S$ respectively then $u \cap s$ consists of at most one point. 
Proof. Suppose $x, y \in u \cap s$ and $x \neq y$. By (1.4) $x$ has a product neighborhood $N$ which does not contain $y$. From the elementary properties of foliations (see [6]) it follows that $V_{s}=\{z \mid s(z) \cap N \neq \varnothing\}$ and $V_{u}=\{z \mid u(z) \cap N \neq \varnothing\}$ are open. $V_{s} \cap V_{u}$ is a neighborhood of $y$. Since $N W(f)=T^{n}$, the periodic points of $f$ are dense in $T^{n}$, (a proof of this due to M. Hirsch is given in (1.7) of [3]). Consequently there is a point $y^{\prime}$ which is in $V_{s} \cap V_{u}-\operatorname{cl}(N)$ and such that $P\left(y^{\prime}\right)$ is a periodic point of $f$. Since $u\left(y^{\prime}\right) \cap N \neq \varnothing$ and $s\left(y^{\prime}\right) \cap N \neq \varnothing$ it follows that $u\left(y^{\prime}\right) \cap s\left(y^{\prime}\right)$ contains a point $x^{\prime}$ in $N$. Hence $u\left(y^{\prime}\right)$ and $s\left(y^{\prime}\right)$ intersect in two distinct points $x^{\prime}$ and $y^{\prime}$.

Suppose $P\left(y^{\prime}\right)$ has period $n$ and let $g=f^{n}$, then $P\left(y^{\prime}\right)$ will be a fixed point of $g$. If we make $P\left(y^{\prime}\right)$ the base point of $T^{n}$ and $y^{\prime}$ the base point of $R^{n}$ we can obtain $\bar{g}$, a covering of $g$, such that $g\left(y^{\prime}\right)=y^{\prime}$. The foliations induced by $g$ are identical with $U$ and $S$. Moreover $u\left(y^{\prime}\right)$ is the unstable manifold and $s\left(y^{\prime}\right)$ the stable manifold of $y^{\prime}$ with respect to $\bar{g}$ and hence $x^{\prime}$ is a homoclinic point of $\bar{g}$. It is not difficult to show that this implies that $x^{\prime}$ is a nonwandering point of $\bar{g}$ (see (7.2) of Smale's paper [7]). However, from (1.7) of [3] we know that periodic points of $\bar{g}$ are dense in $N W(\bar{g})$ so there must be a periodic point $z_{0}$ of $\bar{g}$ which is not equal to $y^{\prime}$. If $m$ is the period of $z_{0}$ then $\bar{g}^{m}\left(y^{\prime}\right)=y^{\prime}$ and $\bar{g}^{m}\left(z_{0}\right)=z_{0}$. Consequently $g^{m}$ is an Anosov diffeomorphism which satisfies the hypothesis of Lemma (1.2) but not the conclusion. Our original assumption that $x, y \in u \cap s$ has led to a contradiction. Q.E.D.

(1.6) Lemma. If $f: T^{n} \rightarrow T^{n}$ satisfies the hypothesis of Theorem 1 and if $u$ and $s$ are leaves of the foliations $U$ and $S$ respectively, then $u \cap s$ consists of a single point.

Proof. In view of (1.5) it suffices to show $u \cap s \neq \varnothing$. If we let

$$
Q=\{x \mid u(x) \cap s \neq \varnothing\},
$$

then because of the local product structure there is an open neighborhood $O$ of $s$ such that $Q=\{x \mid u(x) \cap O \neq \varnothing\}$. Consequently from the basic properties of foliations (see [6]) $Q$ is an open set. We need only show it is closed to show $Q=R^{n}$.

If $w$ is in the closure of $Q$, then because of the local product structure there is a sequence of points of $Q$, all lying in $s(w)$, which converge to $w$. Let $x$ be an element of this sequence and let $y=u(x) \cap s$. Choose a path $\gamma:[0,1] \rightarrow u(x)$ such that $\gamma(0)=x$ and $\gamma(1)=y$ and a path $\rho:[0,1] \rightarrow s(x)$ such that $\rho(0)=x$ and $\rho(1)=w$.

We wish to construct a function $\theta:[0,1] \times[0,1] \rightarrow R^{n}$ with the following properties:

$$
\theta(r, 0)=\gamma(r) ; \quad \theta(0, t)=\rho(t)
$$

and $\theta(r, t)=s(\gamma(r)) \cap u(\rho(t))$. The construction of this function will complete the proof since $\theta(1,1)$ must be $s \cap u(w)$ and hence we will have shown $w \in Q$. Note that if $\theta$ can be defined it is unique by (1.5).

$\theta(r, t)$ is automatically defined if either $r$ or $t$ is 0 , by the condition $\theta(0, t)=\rho(t)$ and $\theta(r, 0)=\gamma(r)$. Also because of the local product structure the set of $(r, t)$ for 
which $\theta$ can be defined satisfying the conditions above is an open set in $[0,1] \times[0,1]$. Let $t_{0}=\sup \{\hat{t} \mid \theta(r, t)$ is defined for $0 \leqq r \leqq 1$ and $0 \leqq t \leqq \hat{t}\}$ and let

$$
r_{0}=\sup \left\{\hat{r} \mid \theta\left(r, t_{0}\right) \text { is defined for } 0 \leqq r \leqq \hat{r}\right\} .
$$

Let $g: T^{n} \rightarrow T^{n}$ be the automorphism of the torus with the property that $g_{*}=f_{*}: H_{1}\left(T^{n}, R\right) \rightarrow H_{1}\left(T^{n}, R\right)$. By (1.1) there exists a map $h: T^{n} \rightarrow T^{n}$ homotopic to the identity such that $g \circ h=h \circ f$. One checks easily that $h$ must carry stable manifolds of $f$ to stable manifolds of $g$; i.e., if $z$ and $z^{\prime}$ are on the same stable manifold of $f$ then $h(z)$ and $h\left(z^{\prime}\right)$ are on the same stable manifold of $g$. Lifting $h$ to $R^{n}$ we obtain a map $\bar{h}: R^{n} \rightarrow R^{n}$ such that $\bar{g} \circ \bar{h}=\bar{h} \circ \bar{f}$. Since $g$ is an Anosov diffeomorphism it too gives rise to two foliations on $R^{n}$. We will denote by $u(z, f)$ and $u(z, g)$ the leaves through $z$ of the unstable foliations induced by $f$ and $g$ respectively and use similar notation for the stable foliations. Because of the properties of $\bar{h}, u(z, f)=u\left(z^{\prime}, f\right)$ implies $u(\bar{h}(z), g)=u\left(\bar{h}\left(z^{\prime}\right), g\right)$ and similarly $s(z, f)$ $=s\left(z^{\prime}, f\right)$ implies $s(\bar{h}(z), g)=s\left(\bar{h}\left(z^{\prime}\right), g\right) . u(0, g)$ is simply the sum of the eigenspaces of $\bar{g}$ which correspond to eigenvalues whose absolute value is greater than one and $u(z, g)$ is simply the translate of $u(0, g)$ to $z$. The analogous result holds for $s(z, g)$.

Let $J=\left\{(r, t) \mid t<t_{0}\right\}$ then $\bar{h}(\theta(J))$ is bounded because its projection onto $u(0, g)$ along $s(0, g)$ is the projection of the compact set $\bar{h}(\gamma([0,1]))$ and its projection onto $s(0, g)$ along $u(0, g)$ is contained in the projection of the compact set $\bar{h}(\rho([0,1]))$. The purpose of all this has been to show that $\theta(J)$ is bounded which now follows because $\bar{h}$ is proper (see 3.4 of [3]).

Since $\theta(J)$ is bounded we can pick a sequence $\left\{\left(r_{n}, t_{n}\right) \mid r_{n} \leqq r_{n+1}\right\}$ in $J$ converging to $\left(r_{0}, t_{0}\right)$ such that the sequence $\theta\left(r_{n}, t_{n}\right)$ converges in $R^{n}$. Let

$$
v=\lim _{n \rightarrow \infty} \theta\left(r_{n}, t_{n}\right)
$$

and let $N$ be a product neighborhood of $v$. We can assume that the sequence $\left\{\theta\left(r_{n}, t_{n}\right)\right\}$ is contained in $N$. Because of the local product structure it is easy to see that sequence $\left\{\theta\left(r_{1}, t_{n}\right)\right\}$ is contained in $N$ and converges to $u(v) \cap s\left(\theta\left(r_{1}, t_{1}\right)\right)$. Hence $\theta\left(r_{1}, t_{0}\right)=u(v) \cap s\left(\theta\left(r_{1}, t_{1}\right)\right)$. Similarly the sequence $\theta\left(r_{n}, t_{1}\right)$ converges to $s(v) \cap u\left(\theta\left(r_{1}, t_{1}\right)\right)=\theta\left(r_{0}, t_{1}\right)$.

Now let $\left\{\left(r_{n}^{\prime}, t_{n}^{\prime}\right)\right\}$ be any sequence converging to $\left(r_{0}, t_{0}\right)$. Then the sequence $\left\{\left(r_{1}, t_{n}^{\prime}\right)\right\}$ converges to $\left(r_{1}, t_{0}\right)$ and since $\theta\left(r_{1}, t_{0}\right) \in N$, we know $\theta\left(r_{1}, t_{n}^{\prime}\right)$ is in $N$ for sufficiently large $n$. Similarly $\theta\left(r_{n}^{\prime}, t_{1}\right)$ is in $N$ for sufficiently large $n$. And we have

$$
\begin{aligned}
\lim _{n \rightarrow \infty} \theta\left(r_{n}^{\prime}, t_{n}^{\prime}\right) & =\lim _{n \rightarrow \infty} u\left(\theta\left(r_{1}, t_{n}^{\prime}\right)\right) \cap s\left(\theta\left(r_{n}^{\prime}, t_{1}\right)\right) \\
& =u\left(\lim _{n \rightarrow \infty} \theta\left(r_{1}, t_{n}^{\prime}\right)\right) \cap s\left(\lim _{n \rightarrow \infty} \theta\left(r_{n}^{\prime}, t_{1}\right)\right) \\
& =u\left(\theta\left(r_{1}, t_{0}\right)\right) \cap s\left(\theta\left(r_{0}, t_{1}\right)\right)=v .
\end{aligned}
$$

Hence by setting $\theta\left(r_{0}, t_{0}\right)=v$ we can continuously extend $\theta$. This implies that it 
must have been the case that $\left(r_{0}, t_{0}\right)=(1,1)$, that is, that $\theta$ can be defined on all of $[0,1] \times[0,1]$. Q.E.D.

(1.7) COROLlaRY. If $f$ satisfies the hypothesis of Theorem 1 then there is a homeomorphism $\psi: R^{n} \rightarrow R^{k} \times R^{n-k}$ taking each leaf $u$ of $U$ onto a $k$-plane and each leaf $s$ of $S$ onto an $(n-k)$-plane, where $k=\operatorname{dim} E^{u}$.

Proof. Let $u_{0}$ be a leaf of $U$ and $s_{0}$ be a leaf of $S$. Then the map

$$
\psi: R^{n} \rightarrow u_{0} \times s_{0} \text { defined by } \psi(x)=\left(s(x) \cap u_{0}, u(x) \cap s_{0}\right)
$$

is the required homeomorphism. Q.E.D.

(1.8) Proof of Theorem 1. We choose a fixed point of $f: T^{n} \rightarrow T^{n}$ (which exists by (1.3)) as base point and we can even assume without loss of generality that this point is 0 . Since $f_{*}: H_{1}\left(T^{n}, R\right) \rightarrow H_{1}\left(T^{n}, R\right)$ is hyperbolic there is a hyperbolic automorphism of the torus $g: T^{n} \rightarrow T^{n}$ which is homotopic to $f$. By (1.1) there exists a base point preserving map $h: T^{n} \rightarrow T^{n}$ homotopic to the identity such that $g \circ h=h \circ f$. We will show that $h$ is a homeomorphism. If $x$ and $y$ are two points lying in the same leaf $u$ of $U$ then $d(u ; x, y)$ will denote the distance between $x$ and $y$ in the intrinsic metric of $u$; i.e., $d(u ; x, y)=$ inf length of $\gamma$ where $\gamma$ runs through the set of piecewise smooth paths joining $x$ and $y$ and lying entirely in $u . d(s ; x, y)$ is similarly defined when $s(x)=s(y)$.

Let $u_{0}$ be a fixed leaf of $U$ and $s_{0}$ a fixed leaf of $S$ and let $x_{0}=u_{0} \cap s_{0}$. Define

and

$$
B(u ; r)=\left\{x \in u_{0} \mid d\left(u_{0} ; x, x_{0}\right) \leqq r\right\}
$$

$$
B(s ; r)=\left\{x \in s_{0} \mid d\left(s_{0} ; x, x_{0}\right) \leqq r\right\} .
$$

Let $D(r)=\left\{x \mid u(x) \cap s_{0} \in B(s, r)\right.$ and $\left.s(x) \cap u_{0} \in B(u, r)\right\} . D(r)$ is in effect a large product neighborhood of $x_{0}$.

We consider the lifts to $R^{n}$ of the maps $f, g$, and $h$, and obtain maps $\bar{f}, \bar{g}$, and $\bar{h}$ such that $\bar{g} \circ \bar{h}=\bar{h} \circ \bar{f}$. Since $h$ is homotopic to the identity it follows that $\bar{h}$ is surjective. Suppose $z$ and $z^{\prime}$ are two points of $R^{n}$ such that $\bar{h}(z)=\bar{h}\left(z^{\prime}\right)$, then if $z^{\prime \prime}=u(z) \cap s\left(z^{\prime}\right)$ we have $\left.\left.\bar{h}\left(z^{\prime \prime}\right)=u(\bar{h}(z) ; g)\right) \cap s\left(\bar{h}\left(z^{\prime}\right) ; g\right)\right)=\bar{h}(z)$.

Let $K$ be a compact fundamental domain for the covering $P: R^{n} \rightarrow T^{n}$ and choose $r$ sufficiently large that $D(r) \supset \bar{h}^{-1}(K)$ (recall that $\bar{h}$ is proper). There exists an integer lattice point $l$ of $R^{n}$ such that $(h(z)+l) \in K$; i.e., $h(z+l)=h\left(z^{\prime \prime}+l\right) \in K$. Thus $z+l$ and $z^{\prime \prime}+l$ are in $D(r)$. Consequently

$$
\begin{aligned}
d\left(u(z) ; z, z^{\prime \prime}\right) & =d\left(u(z+l) ; z+l, z^{\prime \prime}+l\right) \leqq \mu \\
& =\sup \{d(u(x) ; x, y) \mid x, y \in D(r) \text { and } u(x)=u(y)\} .
\end{aligned}
$$

Since $\mu$ in no way depends on $z$ or $z^{\prime \prime}$ we have shown that for any $y, y^{\prime}$ such that $u(y)=u\left(y^{\prime}\right)$ and $\bar{h}(y)=h\left(y^{\prime}\right)$ it must be the case that $d\left(u(y) ; y, y^{\prime}\right) \leqq \mu$.

On the other hand from (1.2) of [3] we know there exist constants $C>0$ and $0<\lambda<1$ such that

$$
d\left(\bar{f}^{n}(u(z)) ; \bar{f}^{n}(z), f^{n}\left(z^{\prime \prime}\right)\right) \geqq C \lambda^{-n} d\left(u(z) ; z, z^{\prime \prime}\right) .
$$


Choose $n$ sufficiently large that $C \lambda^{-n} d\left(u(z) ; z, z^{\prime \prime}\right)>\mu$ and let $y=\bar{f}^{n}(z), y^{\prime}=\bar{f}^{n}\left(z^{\prime \prime}\right)$. Then $u(y)=u\left(y^{\prime}\right)$ and $d\left(u(y) ; y, y^{\prime}\right)>\mu$. But also

$$
\bar{h}(y)=\bar{h}\left(\bar{f}^{n}(z)\right)=\bar{g}^{n}(\bar{h}(z))=\bar{g}^{n}\left(\bar{h}\left(z^{\prime \prime}\right)\right)=\bar{h}\left(\bar{f}^{n}\left(z^{\prime \prime}\right)\right)=\bar{h}\left(y^{\prime}\right) .
$$

Thus we have contradicted the assumption that there are two distinct points $z, z^{\prime}$ such that $\bar{h}(z)=\bar{h}\left(z^{\prime}\right)$. Since $\bar{h}$ is injective and surjective it is a local homeomorphism, so $h$ is a covering map. Since $h$ is homotopic to the identity it must be a homeomorphism. Q.E.D.

\section{The homotopy type of Anosov diffeomorphisms.}

(2.1) Lemma. If $g: T^{n} \rightarrow T^{n}$ is a toral automorphism covered by the linear map $\bar{g}: R^{n} \rightarrow R^{n}$ and $\bar{g}$ has 1 as an eigenvalue, then the Lefschetz number $L(g)=0$.

Proof. We will produce a map homotopic to $g$ with no fixed points. Since 1 is an eigenvalue of $\bar{g}$, if $I$ is the identity map of $R^{n}$ then $V=$ image $(\bar{g}-I)$ has dimension strictly less than $n$. Let $J$ denote the integer lattice points of $R^{n}$. Choose $b \in R^{n}$ such that $(V+b) \cap J$ is empty (this is possible since otherwise $R^{n}=\bigcup_{j \in J}(V+j)$ ). Let $\bar{g}^{\prime}$ be the affine map $\bar{g}+b \cdot \bar{g}^{\prime}$ covers an affine map $g^{\prime}$ on $T^{n}$. If $g^{\prime}$ has a fixed point $x$ and $\bar{x} \in R^{n}$ covers $x$, then $\bar{g}^{\prime}(\bar{x})-\bar{x} \in J$. However this is impossible since $\bar{g}^{\prime}(\bar{x})-\bar{x}=\bar{g}(\bar{x})-\bar{x}+b \in V+b$. Thus $g^{\prime}$ which is homotopic to $g$ has no fixed points. Q.E.D.

(2.2) Proof of Theorem 2. Suppose first that the expanding bundle of $f, E^{u}$ is orientable and let $F$ be the fixed point set of $f$. By the Lefschetz fixed point theorem (see [2]) the Lefschetz number of $f, L(f)=i_{f}(F)=\sum_{x \in F} i_{f}(x)$ where $i_{f}(x)$ denotes the index of the fixed point $x . i_{f}(x)= \pm 1$ the sign depending only on $\operatorname{dim} E^{u}$ and whether $d f$ preserves or reverses the orientation of $E^{u}$ (see 4.11 of [7]). Hence $|L(f)|=$ number of fixed points of $f$, so $L\left(f^{a}\right) \neq 0$ for some integer $q$ ( $f$ must have periodic points since $\operatorname{Per}(f)$ is dense in $N W(f)$ which is not empty). The same argument shows $L\left(f^{n q}\right) \neq 0$ for any $n>0$.

Suppose now that $f_{*}: H_{1}\left(T^{n}, R\right) \rightarrow H_{1}\left(T^{n}, R\right)$ has an eigenvalue which is a root of unity, then for some $m, f_{*}^{m}$ has 1 as an eigenvalue. Consequently if $g$ is the toral automorphism homotopic to $f^{m a}$ then $g: R^{n} \rightarrow R^{n}$ has 1 as an eigenvalue so by (2.1) $L(g)=0$. This is not possible however since $L(g)=L\left(f^{m q}\right) \neq 0$. Thus

$$
f_{*}: H_{1}\left(T^{n}, R\right) \rightarrow H_{1}\left(T^{n}, R\right)
$$

can have no eigenvalues which are roots of unity.

Suppose now that $E^{u}$ is not orientable. Let $2 I: T^{n} \rightarrow T^{n}$ be the map which takes $x$ to $x+x$, then $2 I$ is a covering of degree $2^{n}$. Let $f^{\prime}: T^{n} \rightarrow T^{n}$ be the lift of $f$ to this covering, so $f \circ 2 I=2 I \circ f^{\prime} . f^{\prime}$ is an Anosov diffeomorphism and its expanding bundle $E^{u}\left(f^{\prime}\right)=(2 I)^{*}\left(E^{u}(f)\right)$. If $w_{1}$ denotes the first Stieffel-Whitney class then $w_{1}\left(E^{u}\left(f^{\prime}\right)\right)=(2 I)^{*}\left(w_{1}\left(E^{u}(f)\right)\right)=2 w_{1}\left(E^{u}(f)\right)=0$ so $E^{u}\left(f^{\prime}\right)$ is orientable. Hence $f_{*}^{\prime}: H_{1}\left(T^{n}, R\right) \rightarrow H_{1}\left(T^{n}, R\right)$ has no eigenvalues which are roots of unity. Since 
$f_{*} \circ(2 I)_{*}=(2 I)_{*} \circ f_{*}^{\prime}$ it follows that $f_{*}$ also has no eigenvalues which are roots of unity. Q.E.D.

\section{REFERENCES}

1. A. Avez, Topologie des diffeomorphismes d'Anosov qui possedent un invariant integral, C.R. Acad. Sci. Paris 265 (1967).

2. A. Dold, Fixed point index and fixed point theorem for Euclidean neighborhood retracts, Topology 4 (1965), 1-8.

3. J. Franks, "Anosov diffeomorphisms" in Global analysis, Proc. Sympos. Pure Math., vol. 14, Amer. Math. Soc. Providence, R. I. (to appear).

4. - Topological conjugacy and the fundamental group, Lecture Notes of the Amer. Math. Soc. Summer Institute on Global Analysis, (1968).

5. M. W. Hirsch and C. Pugh, Stable manifolds for hyperbolic sets, Bull. Amer. Math. Soc. 75 (1969), 149-152.

6. G. Reeb, Sur certaines propriétés topologiques des variétés feuilletées, Actualités Sci. Indust., no. 1183, Hermann, Paris, 1952.

7. S. Smale, Differentiable dynamical systems, Bull. Amer. Math. Soc. 73 (1967), 747-817.

Massachusetts Institute of Technology,

Cambridge, Massachusetts 